\title{
Configuration mixing in strange tetraquarks $Z_{c s}$
}

\author{
Marek Karliner $\circledast^{1, *}$ and Jonathan L. Rosner $\circledast^{2, \dagger}$ \\ ${ }^{1}$ School of Physics and Astronomy, Raymond and Beverly Sackler Faculty of Exact Sciences, \\ Tel Aviv University, Tel Aviv 69978, Israel \\ ${ }^{2}$ Enrico Fermi Institute and Department of Physics, University of Chicago, \\ 5620 South Ellis Avenue, Chicago, Illinois 60637, USA
}

(Received 19 July 2021; accepted 3 August 2021; published 31 August 2021)

\begin{abstract}
The BESIII Collaboration has observed a candidate for a $c \bar{c} s \bar{u}$ tetraquark $Z_{c s}$ at $\left(3982.5_{-2.6}^{+1.8} \pm 2.1\right) \mathrm{MeV}$ and width $\left(12.8_{-4.4}^{+5.3} \pm 3.0\right) \mathrm{MeV}$, while the LHCb Collaboration has observed a $Z_{c s}$ candidate in the $J / \psi K^{-}$channel with mass of $\left(4003 \pm 6_{-14}^{+4}\right) \mathrm{MeV}$ and width $(131 \pm 15 \pm 26) \mathrm{MeV}$. In this paper, we examine the possibility that these two states are distinct eigenstates of a mixing process similar to that which gives rise to two axial-vector mesons labeled by the Particle Data Group [P. Zyla et al., Prog. Theor. Exp. Phys. 6, $083 C 01(2020)] K_{1}(1270)$ and $K_{1}(1400)$. The main point is that on top a $\bar{c} c$ pair the $Z_{c s}$ states have the same light quark content as the $K_{1}$-s. In the compact tetraquark picture, this implies several additional states, analogous to members of the $K_{1}$ nonet. These states have not yet been observed; nor are they required in the molecular approach. Thus, experimental discovery or exclusion of these extra states will be a critical test for competing models of exotic mesons with hidden charm.
\end{abstract}

DOI: 10.1103/PhysRevD.104.034033

\section{INTRODUCTION}

The discovery of mesons with minimal quark configuration $c \bar{c} q \bar{q}^{\prime}$, where $q$ and $q^{\prime}$ are light quarks $u, d$, s, was pioneered by the observation [1] of the $X(3872)$. The quark configuration of this state includes $c \bar{c} u \bar{u}, c \bar{c}$, and some lesser admixtures, while in terms of mesons, it is mainly $D^{0} \bar{D}^{* 0}+$ charge conjugate (c.c.) The spin, parity, and charge-conjugation eigenvalue of this state is $J^{P C}=1^{++}[2]$.

Over the course of the nearly 20 subsequent years, partners of this state have emerged. Within the compact tetraquark model, this implies the existence of a flavor $S U(3)$ nonet whose neutral members have $J^{P C}=1^{++}$, as well as candidates for membership in a $1^{+-}$nonet [3-7]. ${ }^{1}$ Candidates for the strange members of these nonets have been identified. The BESIII Collaboration has observed a candidate for a $c \bar{c} s \bar{u}$ tetraquark $Z_{c s}$ at $\left(3982.5_{-2.6}^{+1.8} \pm\right.$ 2.1) $\mathrm{MeV}$ and width $\left(12.8_{-4.4}^{+5.3} \pm 3.0\right) \mathrm{MeV}$ [8], while the LHCb Collaboration has observed a $Z_{c s}$ candidate in the

\footnotetext{
*marek@tauex.tau.ac.il

rosner@hep.uchicago.edu

${ }^{1}$ Strictly speaking, $1^{++}, 1^{+-}$denotes the neutral member of a nonet in which the light quark-antiquark pair is in a ${ }^{3} P_{1},{ }^{1} P_{1}$ state. We shall label these basis states $(A, B)$.

Published by the American Physical Society under the terms of the Creative Commons Attribution 4.0 International license. Further distribution of this work must maintain attribution to the author(s) and the published article's title, journal citation, and DOI. Funded by SCOAP ${ }^{3}$.
}

$J / \psi K^{-}$channel with mass of $\left(4003 \pm 6_{-14}^{+4}\right) \mathrm{MeV}$ and width $(131 \pm 15 \pm 26) \mathrm{MeV}$ [9].

A number of interpretations of the $Z_{c s}$ candidates have been put forth [3,10-12], reviewed briefly in Ref. [13]. Here, we propose that within the compact tetraquark model these two states are likely to be distinct eigenstates of a mixing process similar to that which gives rise to two axialvector strange mesons labeled by the Particle Data Group [14] $K_{1}(1270)$ and $K_{1}(1400)$. We discuss the system of these two kaons in Sec. II, apply similar methods to the $Z_{c s}$ states in Sec. III, and conclude in Sec. IV.

\section{MIXING OF STRANGE KAONS}

We label quark-antiquark mesons with the notation ${ }^{2 S+1} L_{J}$, where $S=0,1$ is the total quark spin; $L=$ $S, P, D, \ldots$ stands for orbital angular momentum $0,1,2, \ldots ;$ and $J=0,1,2, \ldots$ denotes total angular momentum. The lowest-level quark-antiquark mesons consist of

$$
\begin{gathered}
S \text {-waves: } J^{P C}=0^{-+}={ }^{1} S_{0}, \quad 1^{--}={ }^{3} S_{1} ; \\
P \text {-waves: } J^{P C}=0^{++}={ }^{3} P_{0}, \quad 1^{++}={ }^{3} P_{1}, \\
1^{+-}={ }^{1} P_{1}, \quad 2^{++}={ }^{3} P_{2} .
\end{gathered}
$$

Here, $P= \pm 1$ stands for parity eigenvalue, while $C= \pm 1$ denotes eigenvalue under charge conjugation, which is only a good quantum number for a $q \bar{q}^{\prime}$ pair if $q^{\prime}=q$. 
TABLE I. Lowest-lying nonets of $J^{P}=1^{+}$mesons.

\begin{tabular}{lccccc}
\hline \hline${ }^{2 S+1} L_{J}$ & $J^{P C}$ & $I=1$ & $I=1 / 2$ & $I=0$ & $I=0$ \\
\hline${ }^{3} P_{1}$ & $1^{++}$ & $a_{1}(1260)$ & $K_{1 A}^{\dagger}$ & $f_{1}(1420)$ & $f_{1}(1285)$ \\
${ }^{1} P_{1}$ & $1^{+-}$ & $b_{1}(1235)$ & $K_{1 B}^{\dagger}$ & $h_{1}(1415)$ & $h_{1}(1170)$ \\
\hline \hline
\end{tabular}

${ }^{\dagger} K_{1}(1270)$ and $K_{1}(1400)$ are mixtures of $K_{1 A, B}$ (see the text).

More generally, we shall use the basis labels $(A, B)$ for $\left({ }^{3} P_{1},{ }^{1} P_{1}\right)$, respectively.

The assignment of some of the observed light mesons to the above quantum numbers is given in Table 15.2 of Ref. [15]. The two lightest nonets of $J^{P}=1^{+}$mesons are also listed in Table I.

Nonstrange states, in the limit of isospin conservation, possess a definite $G$-parity, defined as

$$
G \equiv C \exp \left(i \pi I_{2}\right)
$$

which reduces to $G=C(-1)^{I}$ for the neutral member of an isospin multiplet with isospin $I$. However, because the strange quark is heavier than the up and down quarks, the corresponding symmetry based on the $U$-spin and $V$-spin [16] subgroups of $S U(3)_{F}$ does not exist (though an analog of $G$-parity can be formally defined in the $S U(3)_{F}$ symmetry limit [17]).

Evidence for two resonant states with $J^{P}=1^{+}$(axial vector kaons) in the $K \pi \pi$ spectrum was first noted in Ref. [18]. The mass eigenstates arise from mixing between $K_{1 A}={ }^{3} P_{1}$ and $K_{1 B}={ }^{1} P_{1}$, for instance as a result of sharing $K^{*}(892) \pi$ and $K \rho$ decay modes $[19,20]$. They may be written as [19] (Ref. [21] uses a different phase convention)

$$
\left|K_{1}(1270)\right\rangle=\left|K_{1 A}\right\rangle \cos \phi-\left|K_{1 B}\right\rangle \sin \phi
$$

$$
\left|K_{1}(1400)\right\rangle=\left|K_{1 A}\right\rangle \sin \phi+\left|K_{1 B}\right\rangle \cos \phi .
$$

Both $K_{1}(1270)$ and $K_{1}(1400)$ can couple to $K^{*}(892) \pi$ and $K \rho$, in $S$ and $D$ waves. Reference [21] quotes parameters of Ref. [22] for these decays as shown in Table II, while current Particle Data Group (PDG) values including those obtained from nondiffractive axial kaon production are summarized in Table III. The analysis of Ref. [19] favored the range $10^{0}<\phi<35^{0}$. For that range, the mixing between mass eigenstates and basis states $\left|K_{1 A, B}\right\rangle$ is such that $K_{1}(1270)$ couples mainly to $K \rho$ in $S$-wave, with suppressed $S$-wave coupling to $K^{*} \pi$ despite greater phase space, while $K_{1}(1400)$ couples mainly to $K^{*} \pi$ in the $S$-wave. This pattern is qualitatively similar to that in an updated version [20,21]. Mixing of states with similar quantum numbers such that different eigenstates have different decay modes is familiar from many other examples in hadron spectroscopy [23-25].

\section{MIXING OF $Z_{c s}$ STATES}

Within the compact tetraquark model, it is natural to expect that the $Z_{c s}$ candidates at 3982.5 and $4003 \mathrm{MeV}$ are the analogs of $K_{1}(1270)$ and $K_{1}(1400)$ with an extra $c \bar{c}$ pair. The light-quark degrees of freedom are linear combinations of a ${ }^{3} P_{1}$ and a ${ }^{1} P_{1} q \bar{q}^{\prime}$ pair. This scheme is close to a superposition of Solutions 1 and 2 of Ref. [4], in which $Z_{c s}(3982.5)$ and $Z_{c s}(4003)$ are distinct states of ${ }^{2 S+1} P_{J}$ : ${ }^{3,1} P_{1}$ for Solution 1 and ${ }^{1,3} P_{1}$ for Solution 2 . In our solution, it is the unmixed strange ${ }^{3} P_{1}$ state which should have a mass $M_{A}$, which is the average of the nonstrange $X(3872)$ and the $c \bar{c} s \bar{s}$ state called $X(4140)$,

$$
M_{A}=(4146.8+3871.65) / 2 \mathrm{MeV}=4009 \mathrm{MeV},
$$

where we have used the latest PDG masses and the identification by Lebed and Polosa [7] of the $X(4140)$ as a $1^{++} c \bar{c} s \bar{s}$ state. In addition to the $X(3872)$, assumed

TABLE II. Masses and decay properties of axial vector kaons as measured by the ACCMOR Collaboration [22] in diffractive production and quoted in Ref. [21]. A small $D$-wave contribution to $K_{1}(1270) \rightarrow K \rho$ neglected.

\begin{tabular}{lccccc}
\hline \hline$K_{1}$ & $M, \mathrm{MeV}$ & $\Gamma_{K_{1}}, \mathrm{MeV}$ & $\mathcal{B}\left(K^{*} \pi\right)_{S}$ & $\mathcal{B}\left(K^{*} \pi\right)_{D}$ & $\mathcal{B}(K \rho)_{S}$ \\
\hline$K_{1}(1270)$ & $1270 \pm 7$ & $90 \pm 8$ & $0.13 \pm 0.03$ & $0.07 \pm 0.006$ & $0.39 \pm 0.04$ \\
$K_{1}(1400)$ & $1410 \pm 25$ & $165 \pm 35$ & $0.87 \pm 0.05$ & $0.03 \pm 0.005$ & $0.05 \pm 0.04$ \\
\hline \hline
\end{tabular}

TABLE III. Masses and decay properties of axial vector kaons as summarized in Ref. [14], including nondiffractive production. Small $D$-wave contribution to $K_{1}(1270) \rightarrow K \rho$ neglected.

\begin{tabular}{llcccc}
\hline \hline$K_{1}$ & $M, \mathrm{MeV}$ & $\Gamma_{K_{1}}, \mathrm{MeV}$ & \multicolumn{1}{c}{$\mathcal{B}\left(K^{*} \pi\right)_{S}$} & \multicolumn{1}{c}{$\mathcal{B}\left(K^{*} \pi\right)_{D}$} & $\mathcal{B}(K \rho)_{S}$ \\
\hline$K_{1}(1270)$ & $1253 \pm 7$ & $90 \pm 20$ & $0.08 \pm 0.025^{\text {a }}$ & $0.08 \pm 0.025^{\text {a }}$ & $0.42 \pm 0.06$ \\
$K_{1}(1400)$ & $1403 \pm 7$ & $174 \pm 13$ & $0.90 \pm 0.06$ & $0.04 \pm 0.01$ & $0.03 \pm 0.03$ \\
\hline \hline
\end{tabular}

${ }^{\mathrm{a}}$ Neglecting error in $\mathcal{B}\left(K^{*} \pi\right)_{D} / \mathcal{B}\left(K^{*} \pi\right)_{S}=(1.0 \pm 0.7) \%$ 
isosinglet, there should exist a nearby nonstrange isotriplet, whose charged members await detection. Strictly speaking, if we regard the $X(3872)$ as mainly $c \bar{c} u \bar{u}$, thanks to its proximity to the $D^{0} \bar{D}^{* 0}+$ c.c. threshold, there should also exist a state which is mainly $c \bar{c} d \bar{d}$, presumably not far from the $D^{+} \bar{D}^{*-}$ threshold, so the estimate (6) is uncertain by at least several $\mathrm{MeV}$. One should stress that, while the existence of these additional states is a must in the compact tetraquark picture, in the molecular approach, they are not necessary and in some cases downright unlikely.

In particular, $X(3872)$ mass is extremely close, within $0.1 \mathrm{MeV}$ of the $\bar{D}^{0} D^{* 0}$ threshold [26]. One pion exchange likely plays a significant role in $\bar{D}^{0} D^{* 0}$ binding, converting a $\bar{D}^{0} D^{* 0}$ system into a $\bar{D}^{* 0} D^{0}$, which of course has the same mass. On the other hand, in the putative charged partner of $X(3872)$ one pion exchange would have to convert a $\bar{D}^{0} D^{*+}$, with threshold at $3875.1 \mathrm{MeV}$ to a $D^{* 0} D^{+}$, with threshold at $3876.5 \mathrm{MeV}$, a shift of $1.4 \mathrm{MeV}$, much too large in comparison with the dynamics apparently responsible for $X(3872)$ binding.

In view of these conflicting expectations from the compact tetraquark and molecular pictures, an experimental search for the extra states is of very high priority. It can discriminate between these competing models of exotic mesons with hidden charm. A discovery would provide strong support for the compact tetraquark picture, while definite experimental exclusion would be a very strong argument in favor of molecular models.

In what follows, we shall refer to nonets by the lightquark spin-parity ${ }^{(2 S+1)} L_{J}$ of their unmixed members, so we will speak of the ${ }^{3} P_{1}$ and ${ }^{1} P_{1}$ nonets. Regarding decay modes of the ${ }^{3} P_{1}$ nonet, those of the $X(3872)$ and $X(4140)$ are not affected by $K_{1}$-like mixing, because the light quark content of these states is $\bar{u} u$ and $\bar{s} s$, respectively. On the other hand, we propose that as result of the mixing of the ${ }^{1} P_{1}$ and ${ }^{3} P_{1}$ multiplets $Z_{c s}(3982.5)$ is the eigenstate of $c \bar{c} s \bar{u}$ mixing, which couples to $D_{s}^{-} D^{* 0}+D_{s}^{*-} D^{0}$, while $Z_{c s}(4003)$ is the eigenstate which couples to $J / \psi K^{-}$.
Information about the ${ }^{1} P_{1}$ nonet is more sparse. The only firm candidate is the nonstrange isotriplet $Z_{c}(3900)$, whose latest mass listed by the PDG is $(3887.1 \pm 2.6) \mathrm{MeV}$. The $c \bar{c} s \bar{s}$ candidate with $J^{P C}=1^{+-}$is unknown at present. That prevents us from estimating the singly strange member of the nonet with mass $M_{B}$ from the data. If all members of the ${ }^{1} P_{1}$ nonet are $M\left(Z_{c}(3900)\right)-M(X(3872)) \sim 15-20 \mathrm{MeV}$ above those of the ${ }^{3} P_{1}$ nonet, we predict a $C=-1 c \bar{c} s \bar{s}$ candidate around $4160 \mathrm{MeV}$. (See also Refs. [4-7].) It should be able to decay to $D_{s}^{*-} D_{s}^{+}+D_{s}^{-} D_{s}^{*+}$, whose threshold is $4080 \mathrm{MeV}$.

\section{CONCLUSIONS}

We have proposed that the two strange tetraquarks recently observed by the BESIII and $\mathrm{LHCb}$ Collaborations, $Z_{c s}(3982.5)$ and $Z_{c s}$ (4003), are mass eigenstates in which the singly strange light quark degrees of freedom with ${ }^{2 S+1} L_{J}={ }^{3,1} P_{1}$ undergo mixing such that the lighter state couples predominantly to $D_{s}^{-} D^{* 0}+D_{s}^{*-} D^{0}$, while the heavier one couples mainly to the mode in which it was observed, $J / \psi K^{+}$. This mixing is similar to that giving rise to the axial vector kaons $K_{1}(1270)$ and $K_{1}(1400)$, which has been known for more than 50 years. The scheme envisions both states as having $J^{P}=1^{+}$. It predicts a ${ }^{1} P_{1}$ state of negative $C$ around $4160 \mathrm{MeV}$, decaying to $D_{s}^{*-} D_{s}^{+}+D_{s}^{-} D_{s}^{*+}$. The compact tetraquark scheme discussed here involves complete nonets; in a molecular picture, key nonet members [such as the charged partners of the $X(3872)]$ may be absent.

\section{ACKNOWLEDGMENTS}

The research of M. K. was supported in part by NSFCISF Grant No. 3423/19. We thank S. Godfrey for helpful correspondence and R. Lebed for calling our attention to Ref. [5], which also draws the analogy with axial vector kaon mixing but considers $Z_{c s}(3985)$ and $Z_{c s}(4000)$ the same and takes the second state to be $Z_{c s}(4220)$.
[1] S. K. Choi et al. (Belle Collaboration), Observation of a Narrow Charmonium-Like State in Exclusive $B^{ \pm} \rightarrow$ $K^{ \pm} \pi^{+} \pi^{-} J / \psi$ Decays, Phys. Rev. Lett. 91, 262001 (2003).

[2] K. Abe et al. (Belle Collaboration), Experimental constraints on the possible $J^{P C}$ quantum numbers of the $X(3872)$, Report No. BELLE-CONF-0541, arXiv:hep-ex/ 0505038 .

[3] L. Maiani, F. Piccinini, A. D. Polosa, and V. Riquer, Diquark-antidiquarks with hidden or open charm and the nature of X(3872), Phys. Rev. D 71, 014028 (2005).
[4] L. Maiani, A. D. Polosa, and V. Riquer, The new resonances $\mathrm{Z}_{c s}(3985)$ and $\mathrm{Z}_{c s}(4003)$ (almost) fill two tetraquark nonets of broken SU(3) , Sci. Bull. 66, 1616 (2021).

[5] J. F. Giron, R. F. Lebed, and S. R. Martinez, Spectrum of hidden-charm, open-strange exotics in the dynamical diquark model, arXiv:2106.05883.

[6] J. F. Giron and R. F. Lebed, Spectrum of the hidden-bottom and the hidden-charm-strange exotics in the dynamical diquark model, Phys. Rev. D 102, 014036 (2020).

[7] R. F. Lebed and A. D. Polosa, $\chi_{c 0}(3915)$ as the lightest $c \bar{c} s \bar{s}$ state, Phys. Rev. D 93, 094024 (2016). 
[8] M. Ablikim et al. (BESIII Collaboration), Observation of a Near-Threshold Structure in the $K^{+}$Recoil-Mass Spectra in $e^{+} e^{-} \rightarrow K^{+}\left(D_{s}^{-} D^{* 0}+D_{s}^{*-} D^{0}\right)$, Phys. Rev. Lett. 126, 102001 (2021).

[9] R. Aaij et al. (LHCb Collaboration), Observation of new resonances decaying to $J / \psi K^{+}$and $J / \psi \phi$, arXiv: 2103.01803.

[10] Z. Yang, X. Cao, F.-K. Guo, J. Nieves, and M. P. Valderrama, Strange molecular partners of the $Z_{c}(3900)$ and $Z_{c}$ (4020), Phys. Rev. D 103, 074029 (2021).

[11] P. G. Ortega, D. R. Entem, and F. Fernandez, The strange partner of the $Z_{c}$ structures in a coupled-channels model, Phys. Lett. B 818, 136382 (2021).

[12] L. Meng, B. Wang, G.-J. Wang, and S.-L. Zhu, Implications of the $Z_{c s}(3985)$ and $Z_{c s}(4000)$ as two different states, arXiv:2104.08469.

[13] M. Karliner and J.L. Rosner, Comments on new heavy exotic $Z_{c s}$ states, Sci. Bull., https://doi.org/10.1016/j.scib .2021.07.006.

[14] P. Zyla et al. (Particle Data Group), Review of particle physics, Prog. Theor. Exp. Phys. 2020, 083 C01 (2020).

[15] C. Amsler, T. DeGrand, and B. Krusche, Quark model, mini-review No. 15, in Ref. [1].

[16] H. J. Lipkin, Unitary symmetry for pedestrians (or I-spin, U-spin, V-all spin for I-spin), ANL-6942 report (to be published) https://doi.org/10.2172/4658488.
[17] Y. Dothan, Unitary parity, Nuovo Cimento 30, 399 (1963).

[18] G. Goldhaber, Interference Phenomena Associated with Boson Resonance Production, Phys. Rev. Lett. 19, 986 (1967).

[19] E. W. Colglazier and J. L. Rosner, Quark graphs and angular distributions in positive-parity meson decays, Nucl. Phys. B27, 349 (1971).

[20] See the extensive list of early references to this mixing in Ref. [32] of Ref. [19].

[21] A. Tayduganov, E. Kou, and A. Le Yaouanc, The strong decays of $K_{1}$ resonances, Phys. Rev. D 85, 074011 (2012).

[22] C. Daum et al. (ACCMOR Collaboration), Diffractive production of strange mesons at $63 \mathrm{GeV}$, Nucl. Phys. B187, 1 (1981).

[23] R. Koniuk and N. Isgur, Baryon decays in a quark model, Phys. Rev. D 21, 1868 (1980); Erratum, Phys. Rev. D23, 818 (1981).

[24] S. Capstick and W. Roberts, $N \pi$ decays of baryons in a relativized model, Phys. Rev. D 47, 1994 (1993).

[25] M. Karliner and H. J. Lipkin, Possibility of narrow highmass exotic states, arXiv:0710.4055.

[26] R. Aaij et al. (LHCb Collaboration), Study of the lineshape of the $\chi_{c 1}(3872)$ state, Phys. Rev. D 102, 092005 (2020). 